\title{
Expectations of Lumbar Surgery Outcomes among Opioid Users Compared with Non-Users
}

\author{
Marie-Jacqueline Reisener ${ }^{1}$, Alexander P. Hughes ${ }^{1}$, Paul Schadler ${ }^{1}$, Alexa Forman ${ }^{1}$, Oliver C. Sax ${ }^{1}$, \\ Jennifer Shue ${ }^{1}$, Frank P. Cammisa ${ }^{1}$, Andrew A. Sama ${ }^{1}$, Federico P. Girardi ${ }^{1}$, Carol A. Mancuso ${ }^{2}$ \\ ${ }^{I}$ Department of Orthopedic Surgery, Hospital for Special Surgery, New York, NY, USA \\ ${ }^{2}$ Department of Medicine, Hospital for Special Surgery, New York, NY, USA
}

\begin{abstract}
Study Design: Matched cohort study.
Purpose: To compare and describe the effect of opioid usage on the expectations of lumbar surgery outcomes among patients taking opioids and patients not taking opioids.

Overview of Literature: Chronic opioid use is common among lumbar-spine surgery patients. The decision to undergo elective lumbar surgery is influenced by the expected surgery outcomes. However, the effects of opioids on patients' expectations of lumbar surgery outcomes remain to be rigorously assessed.

Methods: A total of 77 opioid users grouped according to dose and duration (54 "higher users," 30 "lower users") were matched 2:1 to 154 non-opioid users based on age, sex, marital status, chiropractic care, disability, and diagnosis. All patients completed a validated 20-item Expectations Survey measuring expected improvement with regard to symptoms, function, psychological well-being, and anticipated future spine condition. "Greater expectations" was defined as a higher survey score (possible range, 0-100) based on the number of items expected and degree of improvement expected.

Results: The mean Expectations Survey scores for all opioid users and all non-users were similar ( 73 vs. $70, p=0.18$ ). Scores were different, however, for lower users (79) compared with matched non-users $(69, p=0.01)$ and compared with higher users $(70, p=0.01)$. In multivariable analysis, "reater expectations" was independently associated with having had chiropractic care $(p=0.03)$, being more disabled ( $p=0.002)$, and being a lower-dose opioid user $(p=0.03)$. Compared with higher users, lower users were also more likely to expect not to need pain medications 2 years after surgery $(47 \%$ vs. $83 \%, p=0.003)$.

Conclusions: Patient expectations of lumbar surgery are associated with diverse demographic and clinical variables. A lower dose and shorter duration of opioid use were associated with expecting more items and expecting more complete improvement compared with non-users. In addition, lower opioid users had greater overall expectations compared with higher users.
\end{abstract}

Keywords: Expectations; Lumbar surgery; Opioids; Lower-back pain

\section{Introduction}

Lower back pain is a common musculoskeletal condition associated with prolonged suffering, deconditioning, and disability [1]. Multiple conservative treatment options exist, including physical therapy and anti-inflammatory

Received Mar 19, 2020; Revised Apr 23, 2020; Accepted Apr 25, 2020

Corresponding author: Alexander P. Hughes

Department of Orthopedic Surgery, Hospital for Special Surgery, 523 East 72nd Street, New York, NY 10021, USA

Tel: +1-212-774-2992, Fax: +1-646-797-8664, E-mail: HughesA@HSS.edu 
medications. For many patients, opioids are used to manage more severe symptoms [2,3]. Opioid costs and opioid prescriptions for spine-related pain have increased significantly in recent years [4]. Over-prescribing of opioids by clinicians has contributed to a substantial opioid epidemic within in the United States, with prescribing rates 2 to 3 times higher than in most European countries [5]. Patients ultimately seek surgery based on the expectation that surgery will ameliorate their condition when other treatment modalities have proven insufficiently effective [6]. Whether the use of opioids before surgery affects expectations toward surgical outcomes remains unknown.

Understanding patient expectations is an important element in the comprehensive preoperative assessment of lumbar surgery patients [7]. Most previous studies used ad-hoc surveys to measure expectations and investigated these expectations as distinct entities, such as "anticipated symptom relief" or "improvement in function" [8,9]. We previously developed and validated a patient-derived survey composed of expectations addressing improvement in symptoms, physical function, psychological well-being, and anticipated future spine condition [10]. In a large cohort of preoperative patients, greater expectations were associated with several variables, including younger age, having had symptoms lasting for a shorter while, having had chiropractic care, worse mood states, and more disability due to pain [11]. The role of opioids was equivocal, which might be reflecting the fact that details regarding dose and duration were not obtained and included in the analysis.

Several recent studies assessed opioid use in spine surgery patients and reported that up to $55 \%$ of the patients take opioids to some degree before surgery [2,3]. In addition to the considerable physical and psychological drawbacks associated with opioids, preoperative use of opioids poses challenges for postoperative pain management and predispose to long-term opioid dependence and worse functional outcomes, such as persistent work disability $[3,12]$. Also, opioid-related changes in brain morphology, cognitive function, and affective symptoms, may impact the patients' perceived perspectives regarding medical care and anticipated outcomes [13,14]. As outlined above, various demographic and clinical variables are associated with patient expectations with regard to lumbar surgery outcomes. The relationship between preoperative opioid use and such expectations, however, has not been rigorously assessed. The purpose of this study was to compare expectations of lumbar surgery in patients with preoperative use of opioids as compared with a sample of matched patients with no preoperative use of opioids. An additional goal was to determine if expectations varied based on the amount of opioids used.

\section{Materials and Methods}

This study was approved by the Institutional Review Board at Hospital for Special Surgery (IRB approval no., 2014-110), and all included patients provided written informed consent. Patients who were taking opioids preoperatively for lumbar symptoms were specifically recruited for this study ("opioid sample"). These patients were then matched with a group of preoperative patients previously recruited during a large survey measuring patient expectations with regard to lumbar surgery (described below). Patients selected for matching were not taking opioids preoperatively ("non-opioid sample"). The recruitment and enrollment processes were the same for both samples, and all patients completed identical surveys regarding demographic and clinical characteristics.

\section{Opioid sample}

Patients were eligible for inclusion in the opioid sample if they were $\geq 18$ years old, were scheduled for lumbar surgery, and were currently taking opioids for low back or radicular pain. Patients were enrolled several days before surgery during routine preoperative office visits. Information about opioid use was obtained from the patients and was described according to type of medication, dose, and frequency and duration of use. Currently, there is no consensus with regard to the definition of chronic preoperative opioid use [15]. Oleisky et al. [15] compared different chronic preoperative opioid use definitions in terms of outcomes after spine surgery. They concluded that combining dosage and duration of opioid usage, like in the Edlund model, would be the best performing model for defining opioid use due to the highest predictive ability for postoperative opioid use and strongest association between preoperative chronic opioid use and 12-month satisfaction and disability [15]. The Edlund model combines duration and dosage in their definition of chronic opioid use (high-dose \& chronic, >91 days; morphineequivalent doses [MED] $>120 \mathrm{mg} /$ day) [16]. Based on the recommendations of Oleisky et al. [15], we combined 
the Schoenfeld model for identifying high-risk patients based on chronic opioid duration ( $>6$ month) and the Edlund definition in our Hospital for Special Surgery's pain management assessment criteria of opioid use using duration and frequency $[16,17]$. Based on these established definitions, we dichotomized opioid-using patients into higher and lower users based on dose and duration. Using standard conversion values, equianalgesic doses were calculated for different types of opioids based on MED expressed as milligrams of morphine per day (mg/ day) [18]. Additionally to Edlund's definition of highdose opioid use ( $>120 \mathrm{mg} /$ day), an MED of $\geq 120 \mathrm{mg} /$ day is associated with an increased risk of overdose and is the threshold value recommended for consultation with pain management experts $[16,18,19]$. By convention, pain for $\geq 3$ months is considered chronic pain. However, similar to Schoenfeld's definition of high-risk patients based on opioid use for $\geq 6$ months, patients with low back pain often undergo surgery after a longer period of conservative treatment, and thus a threshold of $\geq 6$ months has been used to describe long-term opioid use [17,18]. Applying these definitions to our study, we defined higher opioid use as an MED $\geq 120 \mathrm{mg} /$ day or use of opioids at any dose for $\geq 6$ months. Lower use was defined as any opioid use that did not meet these threshold criteria.

At enrollment, patients completed the Hospital for Special Surgery Lumbar Spine Surgery Expectations Survey (Fig. 1), which was developed, tested and validated for as-

Hospital for Special Surgery Lumbar Spine Surgery Expectations Survey

Please circle the number that best describes your response to each question. How much improvement do you expect in the following areas as a result your spine surgery?

\begin{tabular}{|c|c|c|c|c|c|}
\hline & \multirow{2}{*}{$\begin{array}{c}\text { Back to normal or } \\
\text { complete improve- } \\
\text { ment }\end{array}$} & \multicolumn{3}{|c|}{ Not back to normal but... } & \multirow{2}{*}{$\begin{array}{l}\text { I do not have this } \\
\text { expectation. or this } \\
\text { expectation does } \\
\text { not apply to me }\end{array}$} \\
\hline & & $\begin{array}{l}\text { a little } \\
\text { improvement }\end{array}$ & $\begin{array}{c}\text { a moderate } \\
\text { amount of } \\
\text { improvement }\end{array}$ & $\begin{array}{c}\text { a little } \\
\text { improvement }\end{array}$ & \\
\hline Relieve pain & 1 & 2 & 3 & 4 & 5 \\
\hline Relieve symptoms that interfere with sleep & 1 & 2 & 3 & 4 & 5 \\
\hline Improve ability to walk more than several blocks & 1 & 2 & 3 & 4 & 5 \\
\hline Improve ability to stand more than half an hour & 1 & 2 & 3 & 4 & 5 \\
\hline Regain strength in legs & 1 & 2 & 3 & 4 & 5 \\
\hline Improve balance & 1 & 2 & 3 & 4 & 5 \\
\hline Improve ability to go up and down stairs & 1 & 2 & 3 & 4 & 5 \\
\hline Improve ability to manage personal care (such as dress, bathe) & 1 & 2 & 3 & 4 & 5 \\
\hline Improve ability to drive & 1 & 2 & 3 & 4 & 5 \\
\hline Remove need for pain medications & 1 & 2 & 3 & 4 & 5 \\
\hline $\begin{array}{l}\text { Improve ability to interact with others (such as social and fam- } \\
\text { ily activities) }\end{array}$ & 1 & 2 & 3 & 4 & 5 \\
\hline Improve sexual activity & 1 & 2 & 3 & 4 & 5 \\
\hline $\begin{array}{l}\text { Improve ability to perform daily activities (such as chores, shop- } \\
\text { ping, errands) }\end{array}$ & 1 & 2 & 3 & 4 & 5 \\
\hline Improve ability to exercise for general health & 1 & 2 & 3 & 4 & 5 \\
\hline $\begin{array}{l}\text { Remove restrictions in activities (such as be more mobile, not } \\
\text { have to rest every few minutes) }\end{array}$ & 1 & 2 & 3 & 4 & 5 \\
\hline $\begin{array}{l}\text { If currently employed: fulfill job responsibilities (such as work } \\
\text { requires hours, complete expected tasks) }\end{array}$ & 1 & 2 & 3 & 4 & 5 \\
\hline $\begin{array}{l}\text { If currently work-disabled or unemployed due to spine: go back } \\
\text { to work for salaried employment }\end{array}$ & 1 & 2 & 3 & 4 & 5 \\
\hline Reduce emotional stress or sad feelings & 1 & 2 & 3 & 4 & 5 \\
\hline Stop my spine condition from getting worse & 1 & 2 & 3 & 4 & 5 \\
\hline Remove the control my spine condition has on my life & 1 & 2 & 3 & 4 & 5 \\
\hline
\end{tabular}

Fig. 1. The Lumbar Spine Surgery Expectations Survey (reprinted with the permission from the the Hospital for Special Surgery). 
sessment of psychometric properties using a large cohort of clinically diverse patients [10,11,20-22]. The survey is composed of 20 items addressing outcome expectations after recuperation from surgery, defined as after 2 years. Three items specifically address pain, i.e. pain relief, relief of pain that interferes with sleep, and dismissed need for pain medications. Patients were asked how much improvement they expected for each of the 20 items with response options of complete improvement (4 points), a lot of improvement ( 3 points), a moderate amount of improvement ( 2 points), a little improvement (1 point), or not having any of these expectations ( 0 points). The score was calculated as the sum of the patient's points divided by the maximum possible points and could range from 0-100, with higher scores indicating greater expectations. Patients also completed the modified Oswestry Disability Index (ODI) survey, a well-established measure of disability due to low back pain; scores can range from 0-100; the higher the scores, the worse the condition [23]. To assess mental health, patients completed the short-form Geriatric Depression Scale, a validated 15-item scale with a threshold score indicating a positive screen for depression, and the Spielberg State Anxiety Inventory, a validated 20 -item scale with population norms available for comparison [24-26].

\section{Non-opioid sample}

Patients in the matched sample were drawn from the cohort previously assembled to develop the Expectations Survey. These patients were also $\geq 18$ years old, were scheduled for lumbar surgery, and were interviewed during routine preoperative visits. There were 420 patients in this cohort; of these, $45 \%$ were taking opioids, but details necessary to calculate MED had not been not recorded. The remaining 55\% (231 patients) were not taking any opioids and thus constituted an eligible cohort for matching for the current analysis.

Matching was performed according to variables identified during the development of the Expectations Survey to be associated with greater expectations [11]. These criterion variables included younger age, marital status (specifically, not being widowed), having had chiropractic care, having a diagnosis of herniated nucleus pulposus (HNP), and worse ODI scores. Matching was first done by sex and marital status, followed by ODI score within 10 points; then, according to chiropractic care and and age within 5 years (i.e., if 30 years they had to be between $25-$ 35 years). The final matching criterion, i.e., diagnosis, was obtained from surgeons preoperatively and dichotomized as HNP as a more acute issue versus a chronic condition such as degenerative spondylolisthesis or degenerative lumbar scoliosis. Matching was performed per patient with two opioid non-users matched to each opioid user (2:1 match).

\section{Data analysis}

The sample size was based on a projected difference in Expectations Survey score (i.e., the primary outcome) between the opioid-user and the non-user samples. For a difference of 10 points, a power of $80 \%$, and an alpha of $0.05,130$ patients in total were required. To be able to detect any smaller differences in scores, we doubled the matching and included a total of 231 patients.

The first series of analyses compared all opioid users to all non-users for demographic and clinical characteristics using chi-square tests and $t$-tests. The samples were then compared according to the features of the Expectations Survey, including the number of items expected, the mean amount of improvement expected for all items, and the Expectations Survey score. Additional sub-analyses similarly compared higher and lower opioid groups to their respective non-opioid matches. Specifically, separate analyses were performed for higher users versus their matched sample, and lower users versus their matched sample.

The higher users also were compared with the lower users in exploratory analyses. Given the fact that these groups were not matched to each other, multivariable linear regression was performed with the Expectations Survey score as the dependent variable. Independent variables included age, marital status, previous chiropractic care, diagnosis, ODI score, and higher/lower opioid group, and the analysis was controlled for those demographic and clinical variables that differed between the groups (described below). Finally, the amount of improvement expected for the three Expectations Survey items that directly address pain were compared between higher and lower groups with chi-square tests.

\section{Results}

In total, 77 patients taking opioids were enrolled from July 2014 to January 2015; the mean time between enrollment 
and surgery was 10 days. Patient mean age was 59 years, $57 \%$ were men, and most patients were married, college graduates, and not working; almost half of the patients had a history of smoking (Table 1). The prevalence of depression and anxiety symptoms were $57.2 \%$ and $89.1 \%$ in the opioid user group compared to $26.6 \%$ and $37.7 \%$ in the non-user group. Most patients had a chronic spine condition, the mean ODI score was 49 , and the median pain score was 7 . The most frequent opioids taken were oxycodone $(43 \%)$, hydrocodone (22\%), acetaminophen/ oxycodone $(20 \%)$, acetaminophen/hydrocodone $(8 \%)$, hydromorphone (5\%), and tapentadol (4\%); $20 \%$ were using or had used more than one type of opioid, and almost half were also taking non-steroidal anti-inflammatory medications for pain. Based on the definition of higher and lower opioid use applied in this study, 47 patients met the criteria for inclusion in the higher-use group. These patients had a median MED of $38 \mathrm{mg} /$ day (interquartile range [IQR], 15-147 $\mathrm{mg} /$ day) and a median duration of opioid use of 18 months (IQR, 7-36 months); 72\% qualified for the higher group based only on duration, $21 \%$ based on both duration and dose, and $7 \%$ based only on dose. The remaining 30 patients met criteria for the lower group. These patients had a median MED of $23 \mathrm{mg}$ /day (IQR, 15-38 mg/day) and a median duration of use of 1.3 months (IQR, 1.0-2.5 months).

In total, 154 patients not taking opioids were enrolled from the previous cohort of patients for a 2:1 match. These non-users had been enrolled from February 2010 to July 2012, with a mean time between enrollment and surgery of seven days. More than half were taking nonsteroidal anti-inflammatory medications for pain.

The matching strategy was successful in terms of constructing a non-user population sample that was comparable to the opioid user sample in terms of the criterion variables as well as the psychosocial variables (i.e., symptoms of depression and anxiety) (Table 1). Opioid users, however, were more likely not to be working and to report more pain even though they did not report more disability due to pain (i.e., they had ODI scores similar to the non-users).

With respect to outcome expectations, for the entire sample, opioid users were more likely than non-users to expect more items from the Expectations Survey (i.e., 18 versus 16 items), but they were not more likely to expect a larger amount of improvement per item or to have higher Expectations Survey scores (i.e., the primary outcome)
(Table 2).

Sub-analyses according to higher and lower opioid groups, however, revealed some important additional findings. Given the fact that non-users were matched to users individually, it was possible to compare the 47 higher users with their corresponding 94 non-user matches, and the 30 lower users with their 60 non-user matches (Table 1). For the comparison involving the higher-users, there was no difference between opioid users and nonusers with respect to Expectations Survey scores (Table 2). However, there were differences between the lower group and their matched non-users. Specifically, lower users were more likely to expect more items, a larger degree of improvement per item, and to have greater Expectations Survey scores. The difference in scores persisted when controlling for having a history of previous spine surgery $(p=0.02)$, which was more common in the non-user sample.

We also compared the high-use with the low-use opioid groups. Although these groups were not matched, they differed across only a couple of demographic and surgical characteristics. Specially, lower users were more likely to be working, college graduates, and to have a diagnosis of HNP, and were less likely to have had previous spine surgery (Table 1). With respect to expectations, lower users expected more improvement per survey item and had higher Expectations Survey scores (Table 2). Multivariable analysis was then carried out with the Expectations Survey score as the dependent variable and age, sex, marital status, working status, education, and diagnosis as independent variables (previous spine surgery was not included as it was correlated with diagnosis). After stepwise backward elimination, the variables that remained associated with having greater Expectations Survey scores were chiropractic care $(p=0.03)$, worse ODI scores $(p=0.002)$, and being in the lower use group $(p=0.03)$ (Table 3$)$.

Finally, the higher and lower groups were compared with respect to the expectation items directly related to pain; i.e., pain relief, relief of pain interfering with sleep, and dismissed need for pain medications (Fig. 2). The lower use group was more likely to expect greater improvement, including complete improvement, for all items. In contrast, less than half of the higher group expected complete improvement for any item. In particular, $83 \%$ of the patients in the low-use group but only $47 \%$ of those in the high-use group expected not to need any pain medications 2 years after surgery $(p=0.008)$. 


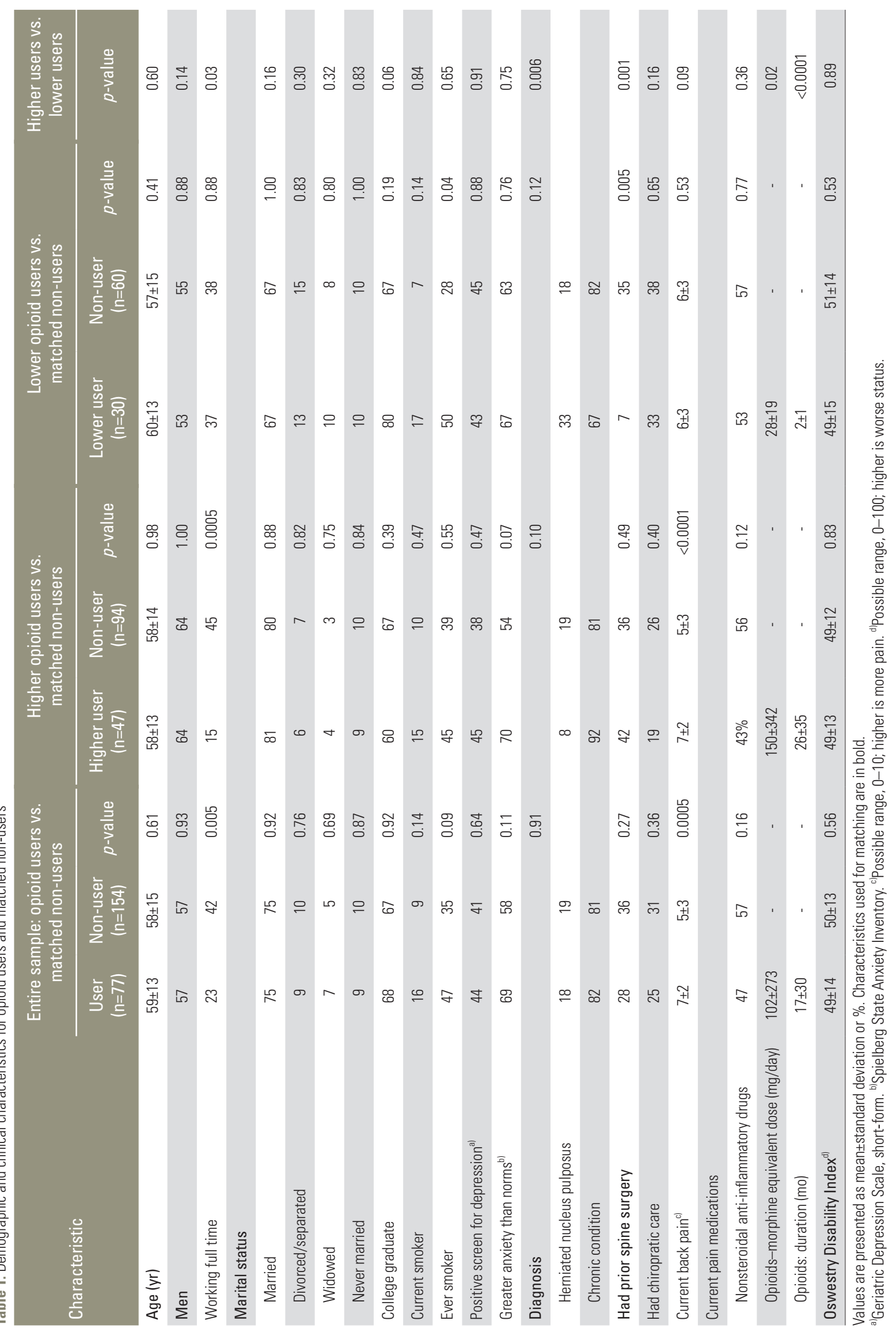



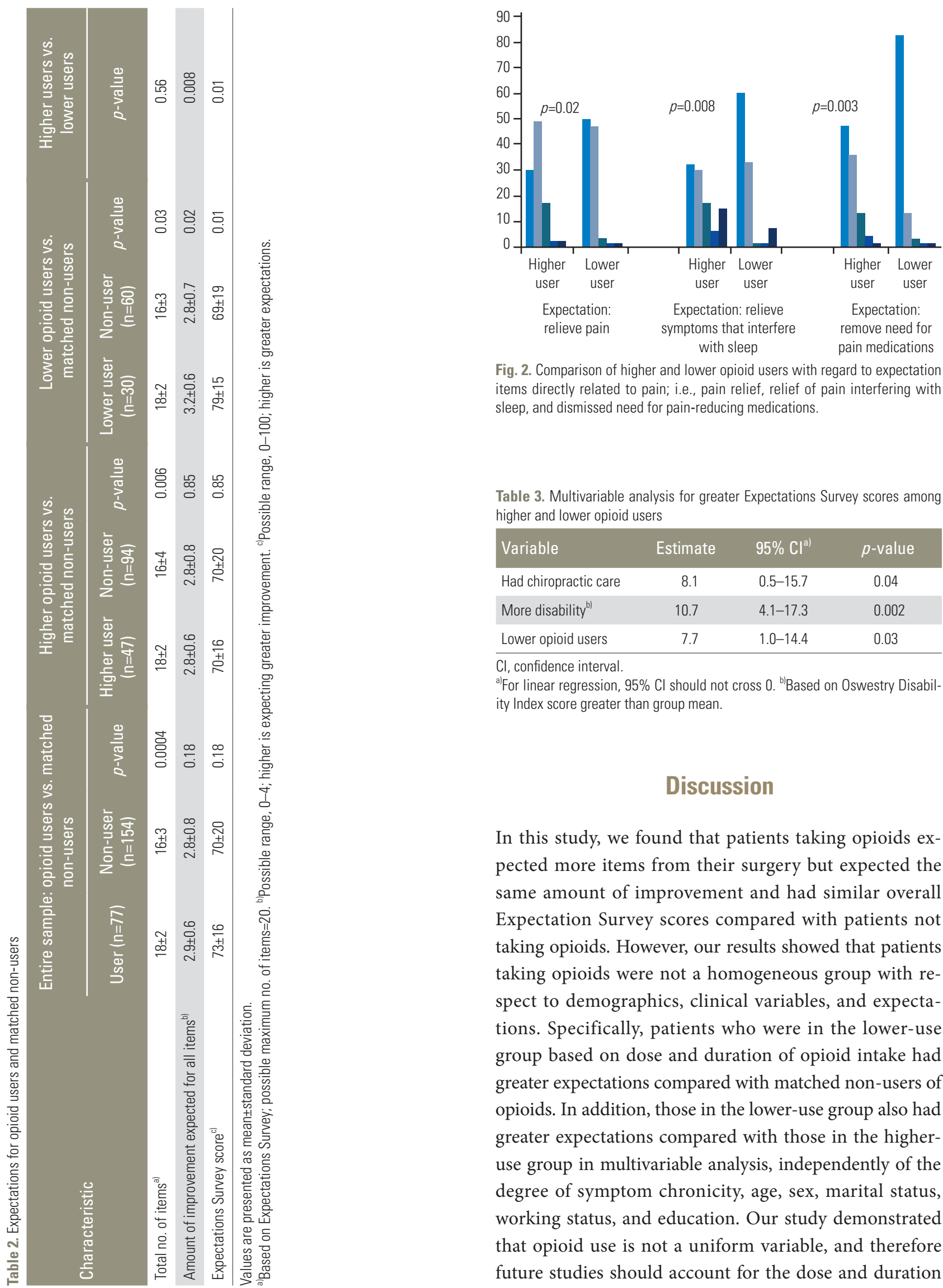

Fig. 2. Comparison of higher and lower opioid users with regard to expectation items directly related to pain; i.e., pain relief, relief of pain interfering with sleep, and dismissed need for pain-reducing medications.

Table 3. Multivariable analysis for greater Expectations Survey scores among higher and lower opioid users

\begin{tabular}{lccc} 
Variable & Estimate & $95 \% \mathrm{Cl}^{\mathrm{a})}$ & $p$-value \\
Had chiropractic care & 8.1 & $0.5-15.7$ & 0.04 \\
More disability $^{\mathrm{b}}$ & 10.7 & $4.1-17.3$ & 0.002 \\
\hline Lower opioid users & 7.7 & $1.0-14.4$ & 0.03 \\
\hline
\end{tabular}

$\mathrm{Cl}$, confidence interval.

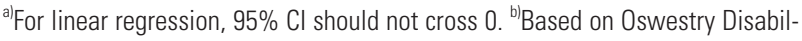
ity Index score greater than group mean.

\section{Discussion}

In this study, we found that patients taking opioids expected more items from their surgery but expected the same amount of improvement and had similar overall Expectation Survey scores compared with patients not taking opioids. However, our results showed that patients taking opioids were not a homogeneous group with respect to demographics, clinical variables, and expectations. Specifically, patients who were in the lower-use group based on dose and duration of opioid intake had greater expectations compared with matched non-users of opioids. In addition, those in the lower-use group also had greater expectations compared with those in the higheruse group in multivariable analysis, independently of the degree of symptom chronicity, age, sex, marital status, working status, and education. Our study demonstrated that opioid use is not a uniform variable, and therefore future studies should account for the dose and duration 
when considering the effect of opioid use on patients' perceived perspectives of surgery.

This study is the first to evaluate the expectations of patients stratified by the extent of opioid use. In a previous study, we demonstrated that any opioid use as significantly associated with the degree of expectations regarding their surgery, but we were unable to tease out the nature of the association, because dose and duration were not measured [11]. In the current analysis, this information was recorded and investigated in detail. In addition, the current analysis focused specifically on the effect of opioids by extensively matching for other variables known to be associated with expectations. Thus, our study is a rigorous evaluation of the relationship between opioid use and patients' expectations toward the outcome of surgery.

Although it might be thought that higher users would have greater expectations because they are likely to be more severely affected by their spine condition, careful examination of the clinical scenario provides possible reasons why we found the opposite to be the case (i.e., lower users had greater expectations). More of the higher users had long-standing degenerative conditions with a dismal outlook, including a high risk of progressive disease. For these patients, the development of residual or recurrent symptoms and disability was likely, and not expecting complete improvement would be appropriate in their situation. In contrast, the lower users had taken to using opioids only relatively recently, and fewer of these had degenerative disease. It is likely that more of the lower users might think that surgery would reverse their condition and that opioid use was only temporary; thus, they had greater expectations. This hypothesis is supported by patient responses to the specific pain medication item in the Expectations Survey, where lower users were more likely to expect complete improvement (i.e., no more need for pain medications) after surgery compared with higher users ( $83 \%$ versus $47 \%)$. Another possible explanation for higher users expecting to continue opioids after surgery derives from current theories about alterations in brain morphology due to chronic opioid exposure, which can result in opioid tolerance, dependence and, in some patients, misuse and addiction [3,14].

Although our findings did not show any significant difference in expectations after lumbar spine surgery in patients who took opioids compared with non-users, management of lumbar spine surgery expectations based on preoperative opioid usage should be considered in the surgeon-patient discussion before surgery $[27,28]$. Previous studies showed that pre-surgical use of opioids is a predictor of various adverse postoperative outcomes in spine surgery such as severe self-rated pain and overall health, and is associated with increased length of hospitalization, surgical complications, and delays in returning to work [29]. Moreover, opioid use before surgery is associated with a considerably higher risk of long-term opioid use and less improvement of the postoperative outcome, and preoperative pain management planning should take these observations into account $[27,30]$.

This study has several limitations. First, patients were enrolled from a tertiary care spine center and their expectations may differ from those of patients in other settings. Furthermore, most opioids had not been prescribed by the spine surgeon; therefore, dose, frequency, and duration were based primarily on the patient's self-reported data. Additionally, the results comparing the expectations of lower and higher opioid users are to be considered preliminary and should be confirmed by studies using larger samples.

Our study showed that patients who take opioids differ by various demographic and clinical variables. Given the fact that these opioid user groups were not matched to each other, differences in sociodemographic factors and clinical conditions of the patients might impact the analysis of the expectations of opioid users and their expectations toward lumbar surgery outcomes and should therefore be considered when interpreting the results.

\section{Conclusions}

In conclusion, patient expectations with regard to the outcome of lumbar surgery are associated with diverse demographic and clinical variables, including the use of preoperative opioids. Lower dose and shorter duration of use were associated with expecting more items and expecting complete improvement for more items compared with opioid non-users matched for multiple relevant variables. In addition, lower opioid users had greater overall expectations compared with higher users. This study demonstrated that opioid users are not a homogeneous sample with respect to expectations. Future studies assessing preoperative opioids should stratify patients according to dose, duration, and potentially other characteristics integral to opioid use such as the extent of expected continued postoperative intake of opioids. 


\section{Conflict of Interest}

No potential conflict of interest relevant to this article was reported.

\section{Funding}

No direct funding was provided for this study. The research reported in this publication was supported by the National Center for Advancing Translational Science of the National Institute of Health under award number UL1TR000457.

\section{References}

1. Rubin DI. Epidemiology and risk factors for spine pain. Neurol Clin 2007;25:353-71.

2. Anderson JT, Haas AR, Percy R, Woods ST, Ahn UM, Ahn NU. Workers' compensation, return to work, and lumbar fusion for spondylolisthesis. Orthopedics 2016;39:e1-8.

3. Armaghani SJ, Lee DS, Bible JE, et al. Preoperative opioid use and its association with perioperative opioid demand and postoperative opioid independence in patients undergoing spine surgery. Spine (Phila $\mathrm{Pa}$ 1976) 2014;39:E1524-30.

4. Martin BI, Turner JA, Mirza SK, Lee MJ, Comstock BA, Deyo RA. Trends in health care expenditures, utilization, and health status among US adults with spine problems, 1997-2006. Spine (Phila Pa 1976) 2009;34:2077-84.

5. Deyo RA, Von Korff M, Duhrkoop D. Opioids for low back pain. BMJ 2015;350:g6380.

6. Deyo RA, Mirza SK. Trends and variations in the use of spine surgery. Clin Orthop Relat Res 2006;443:13946.

7. Mannion AF, Junge A, Elfering A, Dvorak J, Porchet F, Grob D. Great expectations: really the novel predictor of outcome after spinal surgery? Spine (Phila Pa 1976) 2009;34:1590-9.

8. Saban KL, Penckofer SM. Patient expectations of quality of life following lumbar spinal surgery. J Neurosci Nurs 2007;39:180-9.

9. Soroceanu A, Ching A, Abdu W, McGuire K. Relationship between preoperative expectations, satisfaction, and functional outcomes in patients undergoing lumbar and cervical spine surgery: a multicenter study. Spine (Phila Pa 1976) 2012;37:E103-8.

10. Mancuso CA, Cammisa FP, Sama AA, Hughes AP, Ghomrawi HM, Girardi FP. Development and testing of an expectations survey for patients undergoing lumbar spine surgery. J Bone Joint Surg Am 2013;95:1793-800.

11. Mancuso CA, Duculan R, Stal M, Girardi FP. Patients' expectations of lumbar spine surgery. Eur Spine J 2015;24:2362-9.

12. Ciesielski T, Iyengar R, Bothra A, Tomala D, Cislo G, Gage BF. A tool to assess risk of de novo opioid abuse or dependence. Am J Med 2016;129:699-705.

13. Armaghani SJ, Lee DS, Bible JE, et al. Preoperative narcotic use and its relation to depression and anxiety in patients undergoing spine surgery. Spine (Phila Pa 1976) 2013;38:2196-200.

14. Schiltenwolf M, Akbar M, Hug A, et al. Evidence of specific cognitive deficits in patients with chronic low back pain under long-term substitution treatment of opioids. Pain Physician 2014;17:9-20.

15. Oleisky ER, Pennings JS, Hills J, et al. Comparing different chronic preoperative opioid use definitions on outcomes after spine surgery. Spine J 2019;19:984-94.

16. Edlund MJ, Martin BC, Russo JE, DeVries A, Braden JB, Sullivan MD. The role of opioid prescription in incident opioid abuse and dependence among individuals with chronic noncancer pain: the role of opioid prescription. Clin J Pain 2014;30:557-64.

17. Schoenfeld AJ, Belmont PJ Jr, Blucher JA, et al. Sustained preoperative opioid use is a predictor of continued use following spine surgery. J Bone Joint Surg Am 2018;100:914-21.

18. Washington State Agency Medical Directors Group. Interagency guideline on opioid dosing for chronic non-cancer pain: an educational aid to improve care and safety with opioid therapy. Olympia (WA): Washington State Agency Medical Directors Group; 2010 [cited 2019 Dec 3]. Available from: http://www. agencymeddirectors.wa.gov.

19. Dunn KM, Saunders KW, Rutter CM, et al. Overdose and prescribed opioids: associations among chronic non-cancer pain patients. Ann Intern Med 2010;152:85-92.

20. Mancuso CA, Duculan R, Cammisa FP, et al. Fulfillment of patients' expectations of lumbar and cervical spine surgery. Spine J 2016;16:1167-74.

21. Mancuso CA, Duculan R, Cammisa FP, et al. Propor- 
tion of expectations fulfilled: a new method to report patient-centered outcomes of spine surgery. Spine (Phila Pa 1976) 2016;41:963-70.

22. Mancuso CA, Reid MC, Duculan R, Girardi FP. Improvement in pain after lumbar spine surgery: the role of preoperative expectations of pain relief. Clin J Pain 2017;33:93-8.

23. Fairbank JC, Pynsent PB. The Oswestry Disability Index. Spine (Phila Pa 1976) 2000;25:2940-52.

24. Yesavage JA, Sheikh JI. 9/Geriatric Depression Scale (GDS): recent evidence and development of a shorter version. Clin Gerontol 1986;5:165-73.

25. Spielberger CD, Gorsuch RL, Lushene RE, Vagg PR, Jacobs GA. State-Trait Anxiety Inventory for Adults: manual, instrument, and scoring guide. Palo Alto (CA): Consulting Psychologists Press; 1983.

26. Yesavage JA, Brink TL, Rose TL, et al. Development and validation of a geriatric depression screening scale: a preliminary report. J Psychiatr Res 19821983;17:37-49.

27. Sabesan VJ, Petersen-Fitts GR, Sweet MC, Katz DL, Lima DJL, Whaley JD. The impact of preoperative opioid use on outcomes after arthroscopic rotator cuff repair. JSES Open Access 2018;2:155-8.

28. Smith DH, Kuntz J, DeBar L, et al. A qualitative study to develop materials educating patients about opioid use before and after total hip or total knee arthroplasty. J Opioid Manag 2018;14:183-90.

29. Lee D, Armaghani S, Archer KR, et al. Preoperative opioid use as a predictor of adverse postoperative self-reported outcomes in patients undergoing spine surgery. J Bone Joint Surg Am 2014;96:e89.

30. Connolly J 3rd, Javed Z, Raji MA, Chan W, Kuo YF, Baillargeon J. Predictors of long-term opioid use following lumbar fusion surgery. Spine (Phila Pa 1976) 2017;42:1405-11. 